\title{
O "COMPLEXO HISTOLYTICA" E OUTROS PROTOZOARIOS INTESTINAIS EM UM GRUPO DE CRIANÇAS NO RIO DE JANEIRO*
}

\author{
Angela Maria Mazzucco * Wilson Jacinto Silva de Souza * Francisco de Assis \\ Schuster * e Sérgio Gomes Coutinho * *
}

Em um inquérito coprológico realizado em 80 crianças de 1 a 10 anos de idade, residentes em uma área de favela do Rio de Janeiro, foi utilizado o Schaudinn como fixador e o método da hematoxilina férrica para coloração de protozoários intestinais.

Pelo exame de duas preparações de cada amostra de. fezes, evidenciou-se 37 casos $(46,2 \%)$ de infecção pelo "complexo histolytica".

Os cistos e trofozoitos com diâmetro médio superior a 8,5 micra, foram considerados como Entamoeba histolytica e os de diâmetro inferior, como Entamoeba hartmanni.

Encontrou-se 24 casos $(30,0 \%)$ de E. histolytica, sendo que 21 destes (26,3\%) apresentavam também E. hartmanni, e conseqüentemente, havia 3 casos $(3,7 \%)$ de parasitismo isolado pela E. histolytica.

Encontrou-se 34 casos (42,5\%) de E. hartmanni, sendo que 13 deles (16,2\%) apresentavam parasitismo isolado.

Evidenciou-se nesta população examinada, elevada prevalência de "complexo histolytica", com predominância de E. hartmanni.

São relacionados outros protozoários intestinais diagnosticados.

Os autores discutem estes dados e os relacionam com os de outras publicações.

\section{INTRODUÇÃO}

A Entamoeba histolytica, apesar de descrita desde 1875, permanece até hoje com vários de seus aspectos básicos ainda não totalmente esclarecidos.

Kuenen \& Swellengrebel ${ }^{29}$, em 1913, evidenciaram que indivíduos portadores de infecção amebiana assintomática apresentavam na luz intestinal trofozoĺtos de E. histolytica de tamanho pequeno fagocitando apenas bactérias e detritos, a seguir encistando-se e sendo eliminados sem provocar danos.

Ao lado desta "forma minuta" da E. histolytica, Hoare 27 relaciona a chamada "forma magna" da $E$. histolytica, de tamanho maior, invasora dos tecidos, podendo fagocitar hemácias e incapaz de encistar-se, representando uma fase de virulência do parasita que ocorre nos casos de amebíase aguda.

Uma outra ameba, descrita em 1912 por Prowazek $^{46}$ com o nome de Entamoeba hartmanni, tem morfologia semelhante à "forma minuta" da $E$. histolytica, sendo, entretanto, ainda de tamanho menor. Esta $E$. hartmanni não é patogênica, mas seus pequenos cistos têm sido muitas vezes confundidos com os da $E$. histolytica.

A E. hartmanni é aceita como espécie distinta da $E$. histolytica por Bur-

* Trabalho da Disciplina de Parasitologia da Escola de Medicina da Fundação Técnico-Educacional Souza Marques - Rio de Janeiro.

* Monitores ou Estagiários da Disciplina de Parasitologia da Escola de Medicina da Fundação TécnicoEducacional Souza Marques - Rio de Janeiro.

* * Professor Titular.

Recebido para publicação em 11.8.76 
rows ${ }^{10,11,12,14}$, Freedmam \& ElsdonDew $^{21,22}$, Barreto \& Ferriolli ${ }^{7}$, Barretto ${ }^{5,6}$, Ferriolli $^{20}{ }^{\circ}$, Pessôa ${ }^{42}$, Rey ${ }^{4}$ e muitos outros. Todavia, Faust ${ }^{17}$, Hussein ${ }^{28}$, Hajian $^{26}$, entre outros, não admitem a existência da $E$. hartmanni como uma espécie, considerando-a apenas como uma raça pequena da $E$. histolytica.

$E$. hartmanni e $E$. histolytica podem ser muitas vezes confundidas porque ambas possuem cistos quadrinucleados, e segundo Burrows $^{10}$, muitos erros no diagnóstico das espécies do gênero Entamoeba são cometidos, quando se examinam materiais sem coloração ou corados com iodo.

Em virtude das variações de forma (magna e minuta) da $E$. histolytica assim como das possíveis confusões com E. hartmanni, Hoare 27 estabeleceu o chamado "complexo histolytica".

Deste "complexo" fazem parte a $E$. histolytica em suas duas formas:

a) "Magna" - invasora dos tecidos, não se encistando.

b) "Minuta" - comensal e eliminando-se nas fezes, principalmente como cistos.

A $E$. hartmanni, espécie diferente da $E$. histolytica, também pode ser incluída neste complexo, pois muitas vezes é confundida com a "forma minuta" da E. histolytica.

Sapero e cols. ${ }^{48}$. Barretto e cols. ${ }^{8}$, Hus$\operatorname{sein}^{28}$, Barretto \& Ferriolli $^{7}$ entre muitos outros, mediram numerosos cistos e trofozoitos do "complexo histolytica" e verificaram que eles apresentam dimensões variáveis que se inscrevem em uma curva bimodal, isto é, apresentaram dois máximos de frequeência, mostrando que as variações se fazem em torno de dois tamanhos médios, indicando a presença de duas espécies diferentes. A freqüência mínima do tamanho dos cistos vivos encontra-se ao nivel de 10 micra. Foi então estabelecido como limite divisório prático, entre as duas espécies, $E$. histolytica e $E$. hartmanni, o diâmetro médio de 10 micra para os cistos vivos. Para os cistos fixados e corados estabeleceu-se o diâmetro de 9 micra em virtude da retração que sofre o material após a fixação.

Burrows $^{10}$ afirma que a E. hartmanni diferencia-se da $E$. histolytica também por outras características morfológicas evidenciáveis mais nitidamente nas preparações coradas pela hematoxilina férrica.

Pelas publicaçõés que se referem à prevalência do "complexo histolytica" em nosso meio verifica-se que na maioria delas, não é feita a distinção entre $E$. histolytica e $E$. hartmanni englobando-se estas duas espécies sob a denominação de $E$. histolytica. Ainda nestas publicações, em geral, os métodos utilizados não possibilitam uma identificação segura das espécies, porque não se faz, na maioria das vezes, a mensuração dos cistos e a coloração pela hematoxilina férrica.

O presente trabalho foi realizado, para verificar a prevalência de protozoários intestinais, especialmente os do "complexo histolytica" em um grupo de crianças de baixo nível sócio-econômico, no Rio de Janeiro, procurando-se identificar os casos de $E$. histolytica e $E$. hartmanni, estabelecendo a real prevalência da E. histolytica no grupo estudado.

\section{MATERIAL E MÉTODOS}

Foram realizados exames coprológicos em 80 crianças, 37 delas eram do sexo masculino e 43 do sexo feminino, todas na faixa de 1 a 10 anos de idade, residentes em uma área de favela do Rio de Janeiro, no morro do Salgueiro, situado na Tijuca. Não fugindo às características comuns às favelas, a do Salgueiro não possui lixeiras, sendo os detritos lançados em terrenos ou valas abertas. O sistema de esgotos é precário ou inexistente. $O$ fornecimento de água é feito através de um cano geral, de onde os moradores a levam até as suas casas que em sua maioria são de alvenaria sem revestimento, ou de barro batido.

A cada criança foi distribuído um frasco contendo líquido de Schaudinn, para coleta de uma amostra de fezes. De cada amostra assim mantida, foram feitas duas preparações sobre lâminas que após coradas foram examinadas ao microscópio, independentemente, por no mínimo dois dos autores do presente trabalho.

Utilizou-se para a coloração das preparações a hematoxilina férrica, de acordo com a técnica padronizada no Primeiro Encontro de Pesquisadores em Medicina Tropical ${ }^{45}$.

Os cistos e trofozoítos do "complexo histoIytica" fixados pelo Schaudinn e corados pela hematoxilina férrica foram em sua maioria medidos, utilizando-se uma ocular graduada. Aqueles com diâmetro médio superior a 8,5 micra foram considerados como $E$. histolytica e os de diâmetro médio inferior, como $E$. hartmanni. Além deste critério de diferenciação entre estas duas espécies de amebas, baseado no diâmetro dos cistos e trofozoítos também utilizou-se, em determinados casos, critérios baseados nos detalhes morfológicos do cariossoma, cromatina perinuclear e corpos cromatóides quando presentes. 


\section{RESULTADOS}

Das 80 amostras de fezes examinadas para a pesquisa de protozoários intestinais, evidenciou-se pela técnica empregada, 37 casos $(46,2 \%)$ de parasitismo por E. histolytica e/ou E. hartmanni.

O Quadro 1 refere-se à relação destes 37 casos, mostrando que em apenas 3 deles $(3,7 \%)$ houve parasitismo pela $E$. histolytica não acompanhado de infecção pela $E$. hartmanni. Em outros 13 casos $(16,2 \%)$ ocorreu parasitismo pela $E$. hartmanni não acompanhado de $E$. histolytica. Em outros 21 casos $(26,3 \%$ ) evidenciou-se concomitância de parasitismo pelas duas espécies de Entamoeba.

Se relacionar-se o total de casos de parasitismo isolado ou concomitante por $E$. histolytica e $E$. hartmanni verificar-se-á pelo quadro I que foram encontrados um total de 24 casos $(30,0 \%)$ de portadores de E. histolytica e 34 casos $(42,5 \%)$ de indivíduos parasitados pela $E$. hartmanni.

Em relação ao sexo, observou-se que dos 37 casos $(46,2 \%)$ de parasitismo por E. histolytica e/ou $E$. hartmanni, 19 deles eram do sexo feminino e 18 deles do sexo masculino.

Nos 24 casos $(30,0 \%)$ em que havia infecção pela $E$. histolytica somente encontrou-se a "forma minuta", com exceção de apenas 1 caso, em que havia também trofozoítos de grandes dimensões com eritrócitos fagocitados no citoplasma caracterizando assim a "forma magna" da $E$. histolytica.

Os aspectos morfológicos mais comumentes observados no presente trabalho, estão representados nas Figs. 1, 2, 3, 4, 5 e 6.

Nos trofozoitos "forma minuta" da E. histoIytica o cariossoma apresentava-se pequeno e de posição central no núcleo, às vezes subcentral (Fig. 1); a cromatina perinuclear era formada de grânulos pequenos dispostos regularmente na membrana nuclear e freqüentemente encontravam-se bactérias fagocitadas no seu citoplasma.

Os cistos da E. histolytica (Fig. 2 e 3) apresentavam características nucleares semeIhantes às dos seus trofozoitos e os corpos cromatóides quando presentes, eram de grandes dimensões e com as extremidades arredondadas.

Os trofozoítos da E. hartmanni (Fig. 4) freqüentemente possulam características nucleares mais semelhantes às da $E$. coli com um cariossoma grande, excêntrico ou subcentral e a cromatina perinuclear composta de grânulos relativamente grandes com disposição irregular ou em crescente na membrana nuclear. Em menor número de vezes o cariossoma era de posição central no núcleo e a cromatina perinuclear regularmente disposta.

Os cistos da $E$. hartmanni (Figs. 5 e 6 ) possuíam morfologia nuclear semelhante a de seus trofozoítos. Os corpos cromatóides quando presentes, apresentavam-se com morfologia variável, encontrando-se tanto as formas cocóides (Fig. 6) e de bastonetes com as extremidades retas (Fig. 5), como também a forma de pequenos bastonetes com extremidades arredondadas.

Com relação à presença de outros protozoários intestinais no material examinado, os resultados encontram-se no Quadro II.

Ao considerar-se todas as espécies de protozoários intestinais diagnosticadas nas 80 amostras de fezes examinadas, verificou-se que $\mathbf{2 8}$ delas eram negativas.

Nas 52 amostras restantes (65\%) várias delas estavam parasitadas concomitantemente, por 1 ou mais protozoários, verificando-se assim, um total de 162 infecções por protozoários intestinais.

\section{DISCUSSÃO}

\section{1 - "Complexo histolytica"}

Os aspectos morfológicos mais importantes que possibilitam a diferenciação entre $E$. histolytica e E. hartmanni, têm sido discutidos por vários autores, havendo ainda algumas dúvidas e controvérsias.

Segundo Burrows ${ }^{10}$, o trofozoíto de $E$. hartmanni apresenta características morfológicas nucleares, na maioria das vezes diferentes da E. histolytica com o cariossoma relativamente grande e cromatina perinuclear de grânulos grosseiros, irregularmente dispostos. Barretto ${ }^{6}$ encontrou em $78 \%$ dos trofozoítos de $E$. hartmanni examinados, a cromatina perinuclear disposta sob a forma de crescente ou de blocos irregulares, enquanto nos restantes $22 \%$, a cromatina se dispunha de modo contínuo, porém relativamente espessa em relação ao volume nuclear. Só ocasionalmente a disposição da cromatina perinuclear assemelha-se à disposição comumente encontrada na E. histolytica. Por outro lado Gleason e cols. ${ }^{23}$ não observaram qualquer característica morfológica que permitisse distinguir os trofozoítos da $E$. hartmanni e E. histolytica, a não ser os seus tamanhos médios. 
Prevalência das in feccóes concomitantes ou não de $E$. histolytica e $E$. hartmanni, em um grupó de 80 crianças de 1 a 10 anos de idade.

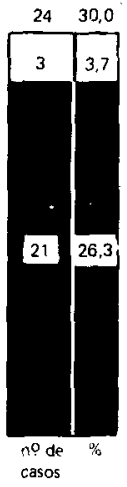

E. histolytica

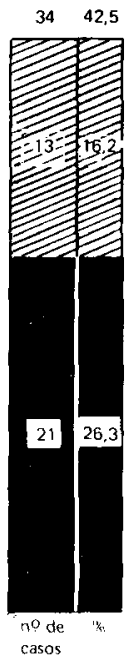

E. nartmanni

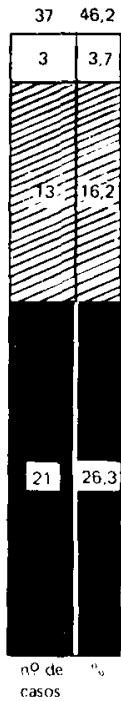
E. histolytica e
$E$ hastmanni 
Prevalència de infeccōes por protozoários intestinais não pertencentes ao "complexo histolytica" em um grupo de 80 criancas de 1 a 10 anos de idade.

nQ̣ de casos

$33.7 \%$

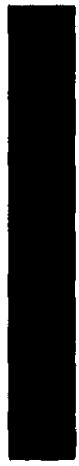

E.n

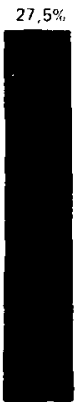

G. 1

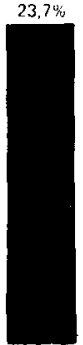

E.c
$23.7 \%$

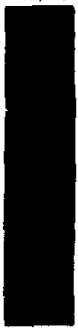

I.b.

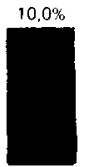

C.m.

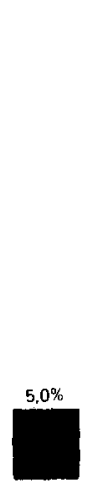

D.f.

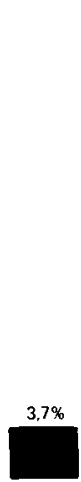

R.i,

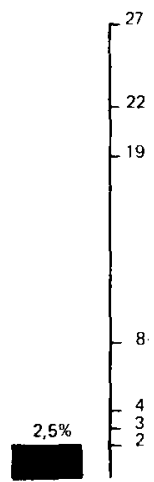

E.h.

E.n. Endolimax nand

C.m. = Chilomastix mesnili

D.f. = Dientamoeba fragilis

R.i. = Retortamonas intestinalis

$\mathrm{E} . \mathrm{h} .=$ Enteromonas hominis

Ec. Entamoeba coll

lodamoeba bütschlii 

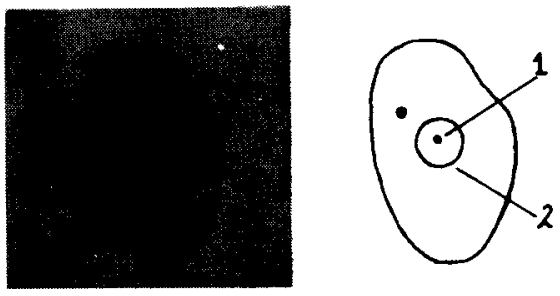

Fig. 1
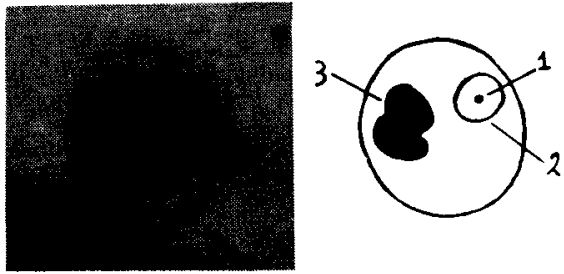

Fig. 3
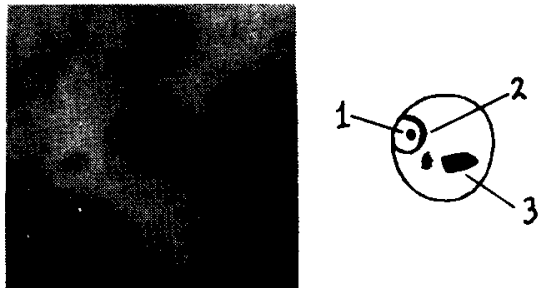

Fig. 5
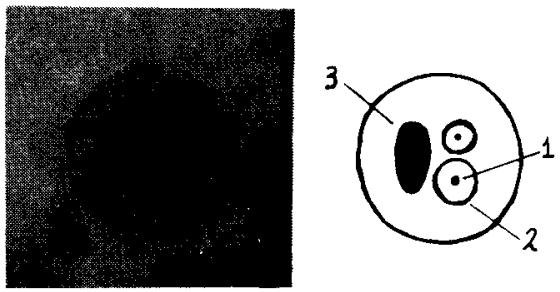

Fig. 2
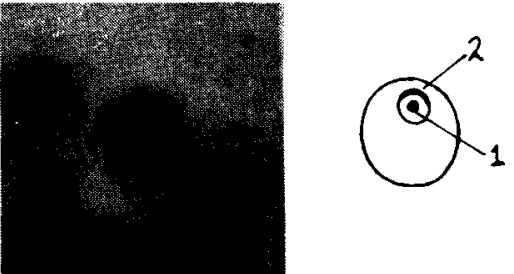

Fig. 4
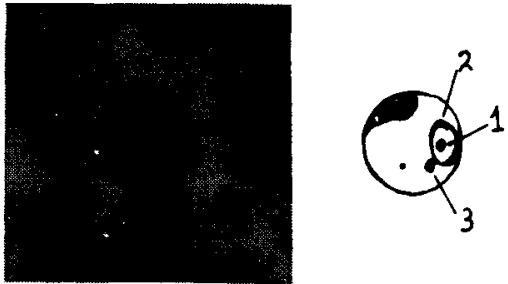

Fig. 6

Fig. 1 - (Fotomicrografia e Desenho) $\times 1650$

Entamoeba histolytica - trofozoito "forma minuta" $(13,3 \times 8,6$ micra)

Núcleo com cariossoma (1) pequeno e subcentral; grânulos de cromatina (2) delicados, dispostos regularmente na membrana nuclear; bactérias fagocitadas no citoplasma.

Fig. 2 - (Fotomicrografia e Desenho) $\times 1650$

Entamoeba histolytica - cisto $(11,3$ micra)

Binucleado, com cariossoma (1) pequeno e central, grânulos de cromatina (2) dispostos regularmente na membrana nuclear; corpo cromatóide (3) de extremidades arredondadas.

Fig 3 - (Fotomicrografia e Desenho) $\times 1650$

Entamoeba histolvtica - cisto $(11,9 \times 10,6$ micra $)$

Uninucleado, cariossoma central (1), com corpos cromatbides (3)

Fig 4 - (Fotomicrografia e Desenho) $\times 1650$

Entamoeba hartmanni - trofozoito $(6,6$ micra $)$

Núcleo com cariossoma (1) grande e subcentral; grânulos de cromatina (2) dispostos em crescente na membrana nuclear.

Fig 5 - (Fotomicrografia e Desenho) $\times 1650$

Entamoeba hartmanni - cisto $(7,3 \times 6,6$ micra)

Uninucleado com cariossoma (1) grande e subcentral; grânulos de cromatina (2) em crescente; corpos cromatóides (3) de extremidades retas.

Fig 6 - (Fotomicrografia e Desenho) $\times 1650$

Entamoeba hartmanni - cisto $(6,6$ micra)

Uninucleado com corpos cromatóides (3) em forma de cocos. 


\section{QUADRO III}

Prevalência do "complexo histolytica" (") segundo vários autores no Brasil

\begin{tabular}{|c|c|c|c|}
\hline Autor & Data & Localidade & "Complexo Histolytica" (*) \\
\hline Young $^{51}$ & 1922 & Amazonas & 29,5 a 23,7 \\
\hline Pessôa \&! Correa ${ }^{41}$ & 1927 & São Paulo & 16,6 \\
\hline Pacheco $^{39}$ & 1928 & Rio de Janeiro & 1,8 \\
\hline Moniz de Aragão ${ }^{35}$ & 1938 & Paraíba & 26,4 \\
\hline Moraes $^{37}$ & 1939 & Santa Catarina & 7 \\
\hline Barcelos $^{4}$ & 1940 & Rio de Janeiro & 19,8 \\
\hline Amaral \& Pires $^{2}$ & 1942 & São Paulo & 39,3 \\
\hline Pontes ${ }^{43}$ & 1945 & Rio de Janeiro & 11,5 \\
\hline Lobo e cols. ${ }^{30}$ & 1952 & Distrito Federal & 6,2 \\
\hline Loures \& Bastos ${ }^{31}$ & 1952 & Distrito Federal & 28,4 \\
\hline Barretto e cols. ${ }^{8}$ & 1960 & São Paulo & 57,62 \\
\hline Coutinho $^{15}$ & 1961 & São Paulo & 26,1 \\
\hline Ferreira e cols. ${ }^{19}$ & 1962 & Guanabara & 4,18 a 14,09 \\
\hline Ferriolli $^{20}$ & 1962 & São Paulo & 19,02 \\
\hline Coutinho ${ }^{16}$ & 1963 & São Paulo & 2,3 \\
\hline Goulart $^{25}$ & 1964 & Guanabara & 0 a 14,4 \\
\hline Martins \& Sampaio ${ }^{32}$ & 1965 & Ceará & 19,7 \\
\hline Wanderley ${ }^{50}$ & 1965 & Rio Grande do Norte & 19,32 \\
\hline Alonso $^{1}$ & 1967 & Minas Gerais & 40,4 \\
\hline Zacharias e cols. ${ }^{52}$ & 1967 & Guanabara & 17,4 \\
\hline Seixas e cols. ${ }^{49}$ & 1968 & São Paulo & 0,3 \\
\hline Araújo $^{3}$ & 1971 & Goiás & 8,95 \\
\hline Fernandes $^{18}$ & 1971 & Rio Grande do Norte & 48,60 \\
\hline Peres e cols. ${ }^{40}$ & 1974 & São Paulo & 1,2 a 2,3 \\
\hline Moretti e cols. ${ }^{38}$ & 1974 & Paraná & 5,12 \\
\hline Mazzucco e cols. & 1976 & Rio de Janeiro & 46,25 \\
\hline
\end{tabular}

$\left(^{*}\right)$ Entamoeba histolytica;

Entamoeba histolytica e/ou Entamoeba hartmanni; raça pequena e/ou raça grande de Entamoeba histolytica. 


\section{QUADRO III}

Prevalência do "complexo histolytica" ( ${ }^{*}$ ) segundo vários autores no Brasil

\begin{tabular}{|c|c|c|c|}
\hline Autor & Data & Localidade & "Complexo Histolytica" (") \\
\hline Young $^{51}$ & 1922 & Amazonas & 29,5 a 23,7 \\
\hline Pessôa \& Correa ${ }^{41}$ & 1927 & São Paulo & 16,6 \\
\hline Pacheco $^{39}$ & 1928 & Rio de Janeiro & 1,8 \\
\hline Moniz de Aragão ${ }^{35}$ & 1938 & Paraíba & 26,4 \\
\hline Moraes 37 & 1939 & Santa Catarina & 7 \\
\hline Barcelos ${ }^{4}$ & 1940 & Rio de Janeiro & 19,8 \\
\hline Amaral \& Pires ${ }^{2}$ & 1942 & São Paulo & 39,3 \\
\hline Pontes ${ }^{43}$ & 1945 & Rio de Janeiro & 11,5 \\
\hline Lobo e cols. ${ }^{30}$ & 1952 & Distrito Federal & 6,2 \\
\hline Loures \& Bastos ${ }^{31}$ & 1952 & Distrito Federal & 28,4 \\
\hline Barretto e cols. ${ }^{8}$ & 1960 & São Paulo & 57,62 \\
\hline Coutinho $^{15}$ & 1961 & São Paulo & 26,1 \\
\hline Ferreira e cols. ${ }^{19}$ & 1962 & Guanabara & 4,18 a 14,09 \\
\hline Ferriolli $^{20}$ & 1962 & São Paulo & 19,02 \\
\hline Coutinho ${ }^{16}$ & 1963 & São Paulo & 2,3 \\
\hline Goulart $^{25}$ & 1964 & Guanabara & 0 a 14,4 \\
\hline Martins \& Sampaio 32 & 1965 & Ceará & 19,7 \\
\hline Wanderley ${ }^{50}$ & 1965 & Rio Grande do Norte & 19,32 \\
\hline Alonso $^{1}$ & 1967 & Minas Gerais & 40,4 \\
\hline Zacharias e cols. ${ }^{52}$ & 1967 & Guanabara & 17,4 \\
\hline Seixas e cols. ${ }^{49}$ & 1968 & São Paulo & 0,3 \\
\hline Araújo $^{3}$ & 1971 & Goiás & 8,95 \\
\hline Fernandes $^{18}$ & 1971 & Rio Grande do Norte & 48,60 \\
\hline Peres e cols. ${ }^{40}$ & 1974 & São Paulo & 1,2 a 2,3 \\
\hline Moretti e cols. ${ }^{38}$ & 1974 & Paraná & 5,12 \\
\hline Mazzucco e cols. & 1976 & Rio de Janeiro & 46,25 \\
\hline
\end{tabular}

(*) Entamoeba histolytica; Entamoeba histolytica e/ou Entamoeba hartmanni; raça pequena e/ou raça grande de Entamoeba histolytica. 
Os cistos da E. hartmanni apresentam núcleos com características semelhantes aos dos trofozoítos e de acordo com Barretto ${ }^{6}$ possuem em geral 3 a 6 corpos cromatóides riziformes ou cocóides. Entretanto, segundo Burrows ${ }^{12}$, nos cistos uninucleados não existem diferenças na distribuição da cromatina nuclear da $E$. hartmanni e da E. histolytica, assim como os corpos cromatóides dos cistos destas duas espécies têm morfologia muito variável, não sendo uma boa características diferencial. Os cistos bi e tetranucleados da $E$. hartmanni possuem núcleos com menor diâmetro do que o dos uninucleados, porém os outros aspectos morfológicos são semelhantes.

Com relação aos trofozoitos de $E$. histolytica mais de $95 \%$ deles apresentam o núcleo com cromatina periférica de distribuição uniforme ou em estreita crescente ${ }^{14}$. O cariossoma muito pequeno é na maioria das vezes central, podendo entretanto apresentar-se subcentral ou excêntrico $0^{10,42,47}$.

Os cistos da E. histolytica apresentam núcleos com caracteres semelhantes aos dos trofozoítos. Os corpos cromatóides, quando presentes, têm em geral a forma de um bastonete grande de extremidades arredondadas ${ }^{42,47}$.

Todas estas características morfológicas possibilitam para estes autores, uma diferenciação entre E. histolytica e E. hartmanni. Entretanto para Sapero e cols. ${ }^{48}$, Barretto e cols. ${ }^{8}$ além de muitos outros, o tamanho dos cistos e trofozoítos permite uma melhor diferenciação entre estas duas amebas.

Em nosso material, utilizamos como principal característica diferencial entre E. histolytica e E. hartmanni o diâmetro médio de cada cisto ou trofozoíto após mais de uma mensuração. Preferimos, entretanto, não utilizar o limite de 9 micra preconizado pela maioria dos autores para cistos fixados e corados, pois nossas observações pessoais evidenciaram uma retração do material fixado, superior a $10 \%$, atingindo em geral a $15 \%$ das dimensões do material examinado a fresco.

Assim sendo, a diferenciação entre $E$. histoIVtica e E. hartmanni, baseada no diâmetro de 8,5 micra, nos pareceu mais precisa e foi o principal critério utilizado. Entretanto nos casos em que os diâmetros médios estavam em torno deste limite de 8,5 micra foram utilizados outros critérios baseados na morfologia do cariossoma, cromatina perinuclear e corpos cromatóides quando presentes (Figs. 1, 2, 3, 4, 5 e 6).
Como todos os exames foram realizados em material corado pela hematoxilina fébrica, estes detalhes morfológicos puderam ser observados com nitidez, ao contrário do que acontece nas preparações a fresco ou coradas pelo lugol.

Segundo Barreto ${ }^{5}$ a coloração pela hematoxilina férrica, além de permitir um diagnóstico seguro nos casos de infecção por amostras de $E$. histolytica com cistos pequenos, que se distinguem mal dos cistos maiores da E. hartmanni, ainda pode revelar parasitos que passam despercebidos no exame de preparações não permanentes.

A prevalência de infecção pelo "complexo histolytica" observada na presente casuística $(46,2 \%)$ pode ser considerada elevada, se compararmos com os resultados observados por outros autores no Brasil. No quadro III estão resumidos vários destes resultados, sem que se pretendesse, entretanto, fazer uma revisão com. pleta da literatura brasileira no assunto.

Prevalências também elevadas foram descritas por Barretto e cols. ${ }^{8}$ quando examinaram as fezes de alienados mentais internados em hospital, utilizando o método da centrífugoflutuação em sulfato de zinco. Os cistos foram examinados após coloração pelo lugol adicionado de $0,1 \%$ de ácido acético para evitar retração. Os autores não referem $E$. hartmanni, mas sim duas raças, pequena e grande de $E$. histolytica, utilizando como limite para separação de ambas, o diâmetro de 10 micra. Encontraram uma prevalência final de infecção pelas raças de $E$. histolytica de $57,62 \%$.

Fernandes $^{18}$ examinou as fezes de 120 reclusos da Penitenciária de Natal, utilizando o método direto com e sem coloração pelo lugol e o método de Hoffman, Pons e Janer, encontrando $48,6 \%$ de infecção pela $E$. histolytica.

Alonso' examinou 2.316 crianças de clínica particular com idades de 1 a 14 anos, utilizando exame direto em material recentemente eliminado, após concentração em solução de cloreto de sódio. Verificou que o parasita mais freqüen. temente encontrado foi a $E$. histolytica $(40,4 \%$ dos casos\}.

Amaral \& Pires ${ }^{2}$, em sentenciados recolhidos à Penitenciária do Estado de São Paulo, encontraram 39,3\% de exames de fezes positivos para E. histolytica, utilizando o método de centrífugo-flutuação em sulfato de zinco e coloração pelo lugol.

Entretanto Fernandes ${ }^{18}$, Alonso ${ }^{1}$ e Amaral \& Pires $^{2}$ não referem o encontro de $E$. hartmanni, nem diferenciam raças pequena ou grande de E. histolytica. 
Estes resultados, ao lado daqueles por nós encontrados, evidenciam a elevada prevalência do "complexo histolytica", principalmente em determinados grupos populacionais em que predomina um padrão sócio-econômico mais baixo como é o caso de favelados e presidiários.

É possível ainda que o elevado número de casos de infecção pelo "complexo histolytica" por nós diagnosticados tenha ocorrido em virtude de terem sido feitas preparações permanentes (hematoxilina férrica) que puderam ser examinadas detidamente, inclusive por mais de uma vez.

Outros autores, entretanto (quadro III) observaram prevalências muito baixas de $E$. histolytica, como é o caso de Pacheco ${ }^{39}$, Seixas e cols. ${ }^{49}$. Peres e cols. ${ }^{40}$ e Coutinho ${ }^{16}$.

Pacheco ${ }^{39}$ examinou amostras de fezes aparentemente normais, provenientes de zonas suburbanas e rurais do Rio de Janeiro, encontrando $1,8 \%$ de $E$. histolytica.

Seixas e cols. ${ }^{49}$ examinaram 7.181 amostras de fezes pelos métodos direto, Hoffman Pons e Janer e o de Willis, encontrando $0,3 \%$ de $E$. histolytica.

Peres e cols. ${ }^{40}$ utilizaram também os métodos de Hoffman Pons e Janer e o de Willis.

É possível que as baixas prevalências eviden. ciadas por estes autores possam estar relacionadas com as técnicas empregadas, pouco apropriadas para a pesquisa de cistos e trofozoítos de protozoários.

Coutinho $^{16}$ encontrou uma baixa prevalên. cia de infecção amebiana $(2,3 \%)$, utilizando o método de centrífugo-flutuação em sulfato de zinco, ao estudar uma casuística composta de universitários. É possível que as condições sócio-econômicas elevadas deste grupo, possam explicar esta baixa prevalência.

Ferriolli ${ }^{20}$ e Loures \& Bastos ${ }^{31}$ fazem a distinção entre $E$. histolytica e $E$. hartmanni, sendo que Young ${ }^{51}$ e Barretto e cols. ${ }^{8}$ referem a presença de raças pequena e grande de $E$. histolytica, com cistos não submetidos a fixadores, respectivamente, menores e maiores què 10 micra.

Nossos resultados (Quadro I) evidenciaram maior prevalência de $E$. hartmanni $(42,5 \%)$ sobre $E$. histolytica $(30,0 \%)$, sendo entretanto, a diferença de prevalência não muito grande. Ferriolli ${ }^{20}$ apesar de encontrar percentuais menores, também evidenciou maior prevalência de $E$. hartmanni $(12,21 \%)$ sobre $E$. histolytica $(10,88 \%)$ no material por ele examinado.

Young $^{51}$ assim como Barretto e cols. ${ }^{8}$ refe- rem a presença de cistos grandes e pequenos de E. histolytica (maiores ou menores que 10 micra) em número semelhante de casos. Entretanto encontraram uma prevalência de eliminadores de cistos grandes em pouco maior que de eliminadores de cistos pequenos.

Por outro lado Loures \& Bastos ${ }^{31}$ encontraram resultados não concordantes com os acima relacionados, em que $\mathbf{2 6 , 8} \%$ dos casos são de portadores de $E$. histolytica e $1,6 \%$ de portadores de E. hartmanni.

Young $^{51}$, Barreto e cols. ${ }^{8}$, Ferriolli ${ }^{20}$, assim como o presente trabalho (Quadro I) evidenciaram co-parasitismo entre $E$. histolytica e $E$. hartmanni, ou eliminação no mesmo paciente de cistos grandes e pequenos de E. histolytica, como preferiam denominar Barretto e cols. ${ }^{8} \mathrm{e}$ Young ${ }^{51}$.

Entretanto, em todas estas publicações também foram encontrados casos de parasitismo por apenas um dos tipos de amebas. Assim sendo, no caso de eliminadores de apenas cistos pequenos, Young ${ }^{51}$ os encontrou entre $9,6 \%$ e $11,5 \%$ de seus casos e Barretto e cols. ${ }^{8}$ em $20,7 \%$ de seus casos. Ferriolli ${ }^{20}$ encontrou $8,14 \%$ de eliminadores de $E$. hartmanni não acompanhada de parasitismo por $E$. histolytica, e no presente trabalho (Quadro I) este fato foi demonstrado em $16,2 \%$ dos casos.

Estas prevalências relativamente elevadas de infecções por $E$. hartmanni ou raça pequena de E. histolytica segundo alguns autores, mostram a importância no diagnóstico laboratorial de amebíase, de diferenciar-se $E$. histolytica e $E$. hartmanni, principalmente pela mensuração dos cistos eliminados. Caso contrário o diagnóstico puro e simples de $E$. histolytica em todos os casos de eliminadores de cistos tetranucleados, estaria englobando uma percentagem variável de casos de parasitismo por E. hartmanni que, como se sabe, não é patogênica.

Em apenas um caso, foi encontrada a forma "magna", invasora dos tecidos da E. histolytica, o que evidencia uma prevalência muito baixa na população ora examinada.

Autores de outros países como Brooke e cols. ${ }^{9}$ encontraram $E$. hartmanni em $11,2 \%$ e $E$. histolytica em $3,4 \%$ dos casos examinados. Sapero e cols. ${ }^{48}$ referem o encontro da raça pequena de E. histolytica em $63 \%$ e raça grande de E. histolytica em $43,3 \%$ dos casos. Estes resultados vêm demonstrar também a maior prevalência da $E$. hartmanni ou raça pequena da E. histolytica.

Por outro lado Meerovitch \& Eaton ${ }^{34}$ encontraram maior prevalência de $E$. histolytica 


\section{$(30,9 \%)$ sobre a $E$. hartmanni $(6,2 \%)$.}

Burrows ${ }^{17}$ relacionando resultados de vários autores na América do Norte, refere parecer existir uma prevalência discretamente maior de $E$. histolytica no Sul dos EEUU sobre $E$. hartmanni, enquanto que esta última é mais prevalente no Alasca, Canadá e norte dos EEUU.

$\mathrm{Na}$ Índia, parece que o parasitismo por $E$. hartmanni é bem menos freqüente se considerarmos que Prakash \& Tandon ${ }^{44}$ encontraram este protozoário em apenas $1,6 \%$ dos casos e Mathur \& $\mathrm{Kaur}^{33}$ não a encontraram em nenhum caso.

\section{1 - Outros protozoários intestinais}

A prevalência da Giardia lamblia por nós encontrada: $27,5 \%$ (Quadro (I)) pode ser considerada elevada, devendo este dado ser valorizado em virtude da conhecida ação patogênica deste protozoário, principalmente em crianças.

Coutinho ${ }^{15,16}$. Seixas e cols. ${ }^{49}$, Pontes ${ }^{43}$ além de outros autores, encontraram prevalências de infecção por este flagelado de respectiva mente $15,1 \% ; 5,3 \% ; 8 \%$ e $11,5 \%$.

Outros protozoários intestinais diagnosticados na presente casuística, são em geral considerados não patogênicos.

O Quadro II mostra uma prevalência variáve| segundo a espécie, mas predominando Endolimax nana $(33,7 \%)$. Dados semelhantes a estes foram obtidos por Moretti e cols ${ }^{38}$. $(31,62 \%)$. No entanto prevalências maiores foram encontradas por Fernandes ${ }^{18}(63,10 \%)$, Moraes ${ }^{36}$ (43\%) e menores por Zacharias e cols. ${ }^{52}$
$(6,7 \%)$, Wanderley ${ }^{50}(4,2 \%)$ e Seixas e cols. ${ }^{49}$ $(0,8 \%)$.

Também em relação às outras espécies de protozoários diagnosticados, inúmeros autores têm encontrado resultados que demonstram uma prevalência muito variável, como é o caso de Martins \& Sampaio ${ }^{32}$ Fernandes $^{18}$, Moretti e cols. $^{38}$, Lobo e cols. ${ }^{30}$, Gonçalves e cols. ${ }^{24} \mathrm{e}$ Seixas e cols. ${ }^{49}$

Este fato deve estar relacionado principalmente com as diferentes técnicas de diagnóstico empregadas, assim como a variedade das populações examinadas.

Finalmente, pelo exame coproscópico de $\mathbf{8 0}$ crianças residentes em uma área de favela, ficou demonstrado que $65 \%$ delas estavam parasitadas por protozoários intestinais, evidenciando as más condições sanitárias da população. Por outro lado ficou também demonstrado o eleva do risco que correm de adquirirem infecções por protozoários reconhecidamente patogênicos como E. histolytica e Giardia lamblia. Deve-se ainda salientar a elevada prevalência de parasitismo por $E$. hartmanni na população examinada, já que esta ameba tem sido freqüentemente confundida $\operatorname{com} E$. histolytica.

\section{AGRADECIMENTOS}

Os au tores agradecem à Prof. Leila de Souza Nunes pelos trabalhos de fotomicrografias realizados com o apoio da Disciplina de Histologia da Escola de Medicina da Fundação TécnicoEducacional Souza Marques, Serviço do Prof. George B. Doyle Maia.

\section{SUMMARY}

In a coprological survey amongst 80 children from 1 to 10 years old, living in a slum area of Rio de Janeiro, it was used the Schaudinn fixation and the iron hematoxylim stain for intestinal protozoa.

For each faecal sample, two microscopic slides were done and through them, 37 cases $(46,2 \%)$ of infections by "histolytica complex" became evidente.

The cysts and trophozoites of this complex were considered as Entamoeba histolytica when having an average diameter of more than 8.5 micra, and as Entamoeba hartmanni when having an average diameter inferior to that.

There were in 24 cases (30.0\%) of E. histolytica; 21 cases (26.3\%) of which presenting also E. hartmanni and 3 cases (3.7\%) presenting isolated parasitism by $\mathrm{E}$. histolytica.

In 34 cases (42.5\%) of E. hartmanni, 13 cases (16.2\%) of isolated parasitism were detected.

This survey shows a high prevalence of "histolytica complex", with E. hartmanni predominance.

Other diagnosed intestinal protozoa are also related in this survey.

The presented data are discussed by the authors in comparison to other publications on the subject. 


\section{REFERÊNCIAS BIBLIOGRÃFICAS}

1. ALONSO, M.T. - Incidência de protozooses e helmintoses intestinais em crianças no Triângulo Mineiro. Hospital, 72:935, 1967.

2. AMARAL, A.D.F. \& PIRES, C.D.A. Nota sobre a incidência de portadores de cistos de "Endamoeba histolytica". Hospital, 22:411, 1942.

3. ARAÚJO, O.A. - Parasitoses intestinais em quatro municípios goianos. Rev. Goiana Med., 17:49, 1971.

4. BARCELOS, V.F. - Um surto de disenteria amebiana na ilha do Carvalho, em Neves. Arq. Hig. Dep. Nac. Saúde, 10:109, 1940.

5. BARRETTO, M.P. - Diagnóstico da amebíase intestinal pelo exame coprológico. Rev. Inst. Med. trop. São Paulo, 4:269, 1962.

6. BARRETTO, M.P. - Entamoeba histolytica, Schaudinn, 1903 e Entamoeba hartmanni (Prowazek, 1912). Arq. Hig. Saúde Púb., 28:289, 1963.

7. BARRETTO, M.P. \& FERRIOLLI FILHO, F. - Correlação entre o tamanho dos cistos e dos trofozoitas da "Entamoeba histoly. tica" Schaudinn, 1903 e da "Entamoeba hartmanni" Prowazek, 1912. Rev. Brasil. Biol., 22:137, 1962.

8. BARRETTO, M:P., ZAGO FILHO, H. \& AVELINO SILVA, G. - Ocorrência de duas "raças" de "Entamoeba histolytica" distintas pelo tamanho dos cistos. Rev. Brasil. Biol., 20:107, 1960.

9. BROOKE, M.M., HEALY, G.R., LEVY, P., KAISER, R.L. \& BUNCH, W.L. - A sample survey of selected areas in and near Little Rock, Arkansas, to assess the prevalence of Entamoeba histolytica. Bull. WId. HIth. Org., 29:813, 1963.

10. BURROWS, R.B. - Endamoeba hartmanni. Am. J. Hyg., 65:172, 1957.

11. BURROWS, R.B. - Differentiation of Entamoeba hartmanni from the Dwarf Strain of Entamoeba histolytica. Proc. 6th. Cong. Trop. Med. and Mal., 3:360, 1958.

12. BURROWS, R.B. - Morphological differentiation of Entamoeba hartmanni and $E$. polecki from E. histolytica. Amer. J. Trop. Med. Hyg., 8:583, 1959.

13. BURROWS, R.B. - Prevalence of amebiasis in the United States and Canada. Amer. J. Trop. Med. Hyg., 10:172, 1961.

14. BURROWS, R.B. - Identification of Entamoeba hartmanni trophozoites from nuclear structure. Amer. J. Hyg., 79:29, 1964.

15. COUTINHO, J.O. - Nota sobre a prevalência da Entamoeba histolytica em zona rural. Arq. Hig. Saúde Púb., 26:219, 1961.

16. COUTINHO, J.O. - Contribuição ao estudo da epidemiologia da amebíase. Arq. Hig. Saúde Púb., 28:67, 1963.

17. FAUST, E.C. - Parasitologic surveys in Cali, Departamento del Valle, Colombia. I. Incidence and morphologic characteristics of strains of Entamoeba histolytica. Amer. J. Trop. Med. Hyg., 7:4, 1958.

18. FERNANDES, P. - Contribuição ao estudo da prevalência de parasitoses intestinais em reclusos da Penitenciária de Natal, com especial referência à ação terapêutica do WIN - 13146 (Teclozine). Rev. Bras. Med., 28:538, 1971.

19. FERREIRA, L.F., NEGRISOLI, D., AMERICANO DO BRASIL, H., ALONSO D. COUTINHO, S.G. \& RODRIGUES DA SILVA, J. - Incidência de entero-parasitoses em diferentes grupos populacionais do Estado da Guanabara. J. Bras. Med., 6:258, 1962.

20. FERRIOLLI FILHO, F. - Prevalência da Entamoeba histolytica e da Entamoeba hartmanni no município de Ribeirão Preto. São Paulo, (Brasil). Rev. Inst. Med. Trop. São Paulo, 4:305, 1962.

21. FREEDMAN, L. \& ELSDON-DEW, R. Size variation in Entamoeba histolytica. Nature, Lond., 181:433, 1958.

22. FREEDMAN, L. \& ELSDON-DEW, R. Size as a criterion of species in the human intestinal amebae. Amer. J. Trop. Med. Hyg., 8:327, 1959.

23. GLEASON, N.N., GOLDMAN, M. \& CAR VER, R.K. - Size and nuclear morphology of Entamoeba histolytica and Entamoeba 
hartmanni trophozoites in cultures and in man. Amer. J. Hyg., 77:1, 1963.

24. GONCAL VES, A., ANDRADE. J.C.R., GIRIBOLA, L. \& OLIVEIRA, M.C. - Levantamento das parasitoses intestinais e condições sócio-econômicas e sanitárias em um bairro de Botucatu. - S.P., Rev. Soc. Bras. Med. Trop. 7:25, 1973.

25. GOULART, E.G. - Freqüência dos enteroparasitos na infância, em áreas urbanizadas e não urbanizadas (favelas), no Estado da Guanabara. Rev. Bras. Farm., 5:259, 1964.

26. HAJIAN, A. \& BALL, G.H. - Increase in size of Entamoeba hartmanni trophozoites cultured on an enriched medium. Amer. J. Trop. Med. Hyg., 12:709, 1963.

27. HOARE, C.A. - Considérations sur l'étiologie de l'amibiase d'après le rapport hôte - parasite. Bull. Soc. Path. exot., 54:429, 1961.

28. HUSSEIN, Z.H. - The pathogenicity of Entamoeba histolytica I - The mean cyst diameter in large and small race infections. Trans. R. Soc. Trop. Med. Hyg., 55:265, 1961.

29. KUENEN, W.A. \& SWELLENGREBEL, N.H. - Die Entamoeben des Menschen und ihre Praktische Bedeutung - Col. Bkt. (I Orig. I, $71: 378,1913$.

30. LOBO, M.B., MOREIRA, M. \& OLIVEIRA, J.E. - Resultado do exame parasitológico (helmintos e protozoários) de 10.019 amostras de fezes pela Técnica de Faust. Hospital, 42:145, 1952.

31. LOURES, J.C. \& BASTOS, A.F. - Resultados e considerações sobre 250 exames de fezes. Hospital, 42:507, 1952.

32. MARTINS, J.M. \& SAMPAIO, E.M. Enteroparasitoses em uma área do munićpio de Fortaleza I - Alterações hematológicas. Rev. Inst. Med. Trop. São Paulo., 9:388, 1967

33. MATHUR, T.N. \& KAUR, J. - Prevalence of Entamoeba histolytica and other protozoal and helminthic infections in hospital patients in Karnal (Haryana). Indian $J$. Med. Res., 62:1212, 1974.

34. MEEROVITCH, E. \& EATON, R.D.P. Outbreack of amebiasis among indians in North western Saskatchewan, Canada. Amer. J. Trop. Med. Hyg., 14:719, 1965.
35. MONIZ DE ARAGÃO, R. - Febre Tifóide, disenterias e difteria em João Pessoa. Brasil - méd., 58:1058, 1938.

36. MORAES, C.L. - Incidência de parasitoses intestinais em crianças da zona suburbana de Santa Maria, R.S., em 1970. Rev. Fac. Med. - UFSM., 3:33, 1971.

37. MORAES, R.G. - Portadores de quistos de protozoários intestinais entre escolares de Florianópolis - Sta. Catarina - Brasil. Brasil Méd-cirúrg., 1:257, 1939.

38. MORETTI, I.G., CHIEFFI, P.P., NAKAGAWA, E., GOMES, A.C. \& FOIZER, A.C.M. - Contribuição ao estudo da história natural de enteroparasitoses em uma comunidade fechada I - Previencia de enteroparasitas em uma comunidade fechada. Rev. Soc. Bras, Med. Trop., 8:41, 1974.

39. PACHECO, G. - Sur la fréquence des kystes d'amibes et d'autres protozoaires dans des fèces apparemment normales, provenant des zones suburbaines et rurales de Rio-de-Janeiro. Compt. Rend. Soc. Biol., 98:1560, 1928.

40. PERES, M.D., ARTIGAS, P.T. \& LOLLO, N. - Levantamento parasitológico realizado na cidade de Bragança Paulista (Estado de São Paulo), com contribuição ao levantamento da carta planorbídica do Estado de São Paulo. Rev. Soc. Bras. Med. Trop., 7:167, 1973.

41. PESSÔA, S.B. \& CORREA, C. - Cunsiderações acerca de alguns protozoários encontrados nas fezes do homem em São Paulo. Folha Méd., 8:85, 1927.

42. PESSO̊A, S.B. \& MARTINS, A.V. Parasitologia Médica. 9ạ ed., Rio de Janeiro, Ed. Guanabara Koogan, 1974.

43. PONTES, J.P.L. - Incidência da amebíase e da giardíase no Rio de Janeiro. Estudo baseado no exame de 165 indivíduos. Rev. Bras. Med., 2:823, 1945.

44. PRAKASH, O. \& TANDON, B.N. - Intestinal parasites with special reference to Entamoeba histolytica complex, as revealed by routine, concentration and cultural examination of stool specimens from patients with gastro-intestinal symptons. Ind. Jour. Med. Res., 54:10, 1966.

45. PRIMEIRO ENCONTRO DE PESQUISADORES EM MEDICINA TROPICAL Metodologia para avaliação terapêutica de 
drogas antiparasitárias: I - Parasitos Intestinais. São José dos Campos, S. Paulo, 1973.

46. PROWAZEK, S.V. - Weitere Britrag zur Kenntnis der Entamoeben. Arch. Protist. 26:241, 1912.

47. REY, L. - Parasitologia. Ed. Guanabara Koogan, 1973.

48. SAPERO, J.J., HAKANSSON, E.G. \& LOUTTIT, C.M. - The occurrence of two significantly distinct races of Endamoeba histolytica. Amer. J. Trop. Med. Hyg., 22:191, 1942.

49. SEIXAS, A.S.S., MACHADO, H.C., VALL'ALBERT, L. \& ROLLEMBERG,
N.B. - Protozóários e helmintos na cidade de São Carlos (S.P.). Hospital, 73:249, 1968.

50. WANDERLEY, G. - Incidência de protozooses e helmintoses em bancários e familiares na cidade de Natal. Hospital, 68:317, 1965.

51. YOUNG, C.J. - Human intestinal protozoa in Amazonas. Amer. Trop. Med. Parasit., 16:93, 1922.

52. ZACHARIAS, N., GONÇALVES, R.R., PENTEADO, J.F. \& CARVALHO, P.R. Incidência das helmintoses e protozooses na Previdência Social. Hospital, 72:259, 1967. 